\title{
Dermal sheath contraction powers stem cell niche relocation during hair cycle regression
}

\author{
Nicholas Heitman ${ }^{1,2,3}$, Rachel Sennett ${ }^{1,2,3}$, Ka-Wai Mok ${ }^{1,2}$, Nivedita Saxena $^{1,2,3}$, Devika Srivastava ${ }^{1,2}$, \\ Pieter Martino', ${ }^{1,2}$, Laura Grisanti ${ }^{1,2}$, Zichen Wang ${ }^{4}$, Avi Ma'ayan ${ }^{4}$, Panteleimon Rompolas ${ }^{5}$, Michael Rendl, ${ }^{1,2,6 *}$
}

${ }^{1}$ Black Family Stem Cell Institute, Icahn School of Medicine at Mount Sinai, New York, NY, USA. ²Department of Cell, Developmental and Regenerative Biology, Icahn School of Medicine at Mount Sinai, New York, NY, USA. ${ }^{3}$ Graduate School of Biomedical Sciences, Icahn School of Medicine at Mount Sinai, New York, NY, USA. ${ }^{4}$ Department of Pharmacological Sciences, Mount Sinai Center for Bioinformatics, BD2K-LINCS Data Coordination and Integration Center, Knowledge Management Center for Illuminating the Druggable Genome (KMC-IDG), Icahn School of Medicine at Mount Sinai, New York, NY, USA. ${ }^{5}$ Department of Dermatology, Institute for Regenerative Medicine, University of Pennsylvania Perelman School of Medicine, Philadelphia, PA, USA. ${ }^{6}$ Department of Dermatology, Icahn School of Medicine at Mount Sinai, New York, NY, USA. *Corresponding author. Email: michael.rendı@mssm.edu

Tissue homeostasis requires the balance of growth by cell production and regression through cell loss. In the hair cycle during follicle regression, the niche traverses the skin through an unknown mechanism to reach the stem cell reservoir and trigger new growth. Here we identify the dermal sheath that lines the follicle as the key driver of tissue regression and niche relocation via the smooth muscle contractile machinery that generates centripetal constriction force. We reveal that the calcium/calmodulin/myosin light chain kinase pathway controls sheath contraction. When this pathway is blocked, sheath contraction is inhibited, impeding follicle regression and niche relocation. Thus, our study identifies the dermal sheath as smooth muscle that drives follicle regression for reuniting niche and stem cells in order to regenerate tissue structure during homeostasis.

Multipotent progenitors produce new cells to replace those that have been lost by injury or by natural turnover and shedding in rapidly renewing tissues such as the bone marrow and hair follicle (1). However, progenitors have limited renewal capacity and must be replenished from the stem cell reservoir, which is coordinated with specialized niche cells (2-4). During hair growth, the specialized dermal papilla (DP) cluster at the base of follicle bulbs secretes niche signals to orchestrate matrix progenitor proliferation, migration, and differentiation $(5,6)$ (fig. S1A). To reboot the progenitor pool throughout life, repeated cycles of follicle regression (catagen), rest (telogen), and regrowth (anagen) (7) (fig. S1B) derive new cells from the stem cell reservoir located in the bulge and germ of the upper follicle (8-12). At the beginning of regression, matrix progenitors stop proliferating and terminally differentiate (fig. S1B). Throughout regression then nearly all outer root sheath (ORS) progenitors along the entire length of catagen follicles undergo apoptotic pruning (13) (Fig. 1A and fig. S1B) that is driven by extrinsic signals (14, 15). Only the ORS progenitors located closely below the bulge stem cells survive and retain multipotency to give rise to a new bulge and germ $(16,17)$. This new stem cell reservoir then becomes reactivated by niche signals from the intact, surviving DP after it relocated from the follicle base to the new bulge and germ in order to regenerate the follicle of the next growth cycle (18-20) (fig. S1B).

In relocating to the stem cell reservoir, the DP trails the regressing epithelial strand of dying ORS progenitors and travels over $90 \%$ of the hair follicle length (fig. S2) toward the bulge/germ stem cell reservoir. Such drastic tissue remodeling poses a considerable challenge for stem cell regulation in establishing the required proximity to its niche for receiving activating signals. Indeed, detachment of the DP leads to stem cell dysfunction and hair loss $(21,22)$. Two hypotheses for DP movement have been proposed for many years (23): 1) an "apoptotic force" generated by dying cells in the shrinking follicle and 2) a "contraction force" by follicle-encapsulating dermal sheath (DS) cells (Fig. 1A and fig. S1B) based on alpha smooth muscle actin ( $\alpha$ SMA) expression identified nearly 3 decades ago (24).

\section{Dermal sheath is essential for reuniting stem cells and niche}

We set out to address whether the DS is important for niche relocation during follicle regression. We first established DSspecific targeting using cartilage proteoglycan Aggrecan (Acan) as a genetic driver. ACAN protein is found in skin only in the follicle basement membrane that separates the DS from epithelium (25) (Fig. 1B). Using $A c a n^{\text {CreER }}$ mice crossed with the $R 26^{\text {LSL-tdTomato }}$ reporter $\left(\right.$ Acan $\left.^{\text {tdT }}\right)$ enabled inducible targeting during catagen regression, restricted within skin to the DS (fig. S3). To test its functional role, we selectively ablated the DS at early catagen using inducible diphtheria toxin driver $R 26^{L S L-D T A}\left(A_{c a n}{ }^{D T A}\right)$ (Fig. 1C). In follicles with effective ablation, only DS cell remnants were left at the beginning of regression (Fig. 1D). By subsequent telogen rest, follicles in 
control skin were fully regressed (Fig. 1E) and follicles with inefficient DS ablation equally regressed (fig. S4), but many follicles with fully ablated DS (fig. S4) remained aberrantly long (Fig. 1, E and F), suggesting a threshold requirement of minimal remaining DS for regression. In stalled follicles, hair shafts extended down to the follicle base and DPs remained deep in the dermis (Fig. 1G). Stalled follicle lengths ranged from partially regressed to completely stalled deep within the dermis (Fig. 1H). This indicates that the DS is required for progression of follicle regression during catagen and for niche relocation to the stem cell reservoir in the upper follicle.

We next analyzed the stalled follicle phenotype more closely with lineage specific markers (figs. S1A and S5A) to determine the degree of catagen dysfunction in non-regressing hair follicles lacking DS. Immunofluorescence confirmed absence of the DS and widespread ORS cells around shafts of stalled follicles (Fig. 1I). Rounded DP cell clusters at the bulb end were no longer engulfed by proliferative matrix progenitors (Fig. 1I). Likewise, hair shaft and inner root sheath precursors were also absent (fig. S5B), as differentiation during anagen growth (fig. S5A) had ended and catagen regression was initiated. Apoptosis was also undetectable in DS-ablated follicles (Fig. 1I), suggesting that catagen did not proceed beyond the early steps and normal catagen cell death (fig. S5C) was absent. Between hair shafts and ORS was a single differentiated layer (Fig. 1I), reminiscent of the companion layer in growing hair follicles (fig. S5A) and of inner bulge cells at telogen (fig. S5D). Together, the phenotypic analyses indicated that long, non-regressed follicles were not caused by continued matrix progenitor proliferation or erroneous differentiation but resulted from a failure of hair shaft and DP movement toward the skin surface and concomitant absence of ORS progenitor apoptosis. Thus, the DS may exert a physical force fundamental for the upward travel of hair shaft and DP niche.

\section{Dermal sheath expresses the molecular machinery of smooth muscles}

To explore if the DS expresses the contractile molecular machinery of smooth muscles that could execute follicle regression and niche relocation, we isolated DS cells for transcriptome analysis, in comparison to DP and dermal fibroblasts (DF). We flow-sorted DS, DP, and DF populations from cells that were isolated from digested back skins of $S o x 2^{G F P}$;Lef1-RFP reporter mice $(6,26)$, and were also stained for mesenchymal receptor PDGFRA (Fig. 2, A and B, and fig. S6, A and B). DS cells expressed Sox $2^{\text {GFP }}$ and PDGFRA, but not RFP and could be cleanly distinguished from DP and DF (27) (Fig. 2, A and B). Verification of known marker expression for DS, DP, and DF demonstrated their identity and purity (fig. S6C). Analyzing the RNA sequencing-generated transcriptomes of DS, DP, and DF and of ORS, matrix, and melanocytes (27) by hierarchical clustering and principal components established their close lineage relationship (fig. S7, A and B). Comparative analysis of gene expression then revealed a DS molecular signature of 483 enriched genes reflecting their specialized functions (Fig. $2 \mathrm{C}$, fig. S7C, and table S1). Gene ontology analysis of the DS signature expectedly yielded extracellular matrix organization categories (Fig. 2D), as the DS is closely associated with the basement membrane that separates the mesenchyme from follicle epithelium. The DS signature was also enriched for genes involved in "muscle filament sliding" and "smooth muscle contraction" (Fig. 2D). Gene set enrichment analysis for "regulation and process of smooth muscle contraction" (table S2) showed significant enrichment in the DS (Fig. 2E), suggesting smooth muscle identity and function.

Several core components of the $\mathrm{Ca}^{2+}$-dependent smooth muscle contraction pathway (Fig. 2F) were highly enriched in the DS including Calm1 (Calmodulin, CaM), Mylk (Myosin light chain kinase, MLCK), Myh11 (Myosin heavy chain 11), Myl9 (Myosin light chain 9) and Acta2 (aSMA) (Fig. 2G). Several pan-smooth muscle genes (28) such as Cald1 (Caldesmon 1), Smtn (Smoothelin) and Tagln (Transgelin, SM22) were also part of the DS signature (Fig. 2G and fig. S7, D and E). Among those, Cald1 and Smtn are not expressed in contractile myofibroblasts (29), indicating that the DS expresses genes of bona fide smooth muscles. During smooth muscle contraction, mechanical forces are generated through actomyosin cross-bridges and ATP-powered myosin ratcheting action (30) (Fig. 2F). 3-dimensional immunofluorescence revealed that the DS forms a network of $\alpha$ SMA stress fibers that wrap the follicle in concentric rings (Fig. $2 \mathrm{H}$ ) suggesting that the potential actomyosin forces would be directed toward the center of the longitudinal axis in centripetal fashion. Actomyosin cross-bridge formation is promoted when CaM-activated MLCK phosphorylates regulatory myosin light chains (e.g., MYL9; Fig. 2, F, G, and I) that associate with myosin heavy chain molecular motors (e.g., MYH10; Fig. 2, F, G, and I). Expression of phosphorylated pMYL9 confirms the active state of myosin in the entire length of DS (Fig. 2I), which we observed throughout catagen (fig. S8) and supports DS functional contractile activity. Finally, several smooth muscle proteins were expressed in human scalp hair follicles suggesting conservation of smooth muscle components in the DS between mice and humans (fig. S9). Overall, these results demonstrate that the DS harbors the contractile apparatus and its regulatory elements, long hypothesized by the presence of $\alpha$ SMA (24).

\section{Dermal sheath contraction is required for follicle regression}

We next explored whether DS cells can functionally contract 
in a smooth muscle-like fashion through $\mathrm{Ca}^{2+}$-dependent MLCK activation. Isolated, short-term cultured DS cells were membrane depolarized by an extracellular $\mathrm{K}^{+}$spike to activate voltage-gated $\mathrm{Ca}^{2+}$ channels in the presence of Fluo8 fluorescent $\mathrm{Ca}^{2+}$ indicator (fig. S10A). The switch from $\mathrm{Na}^{+}$to high $\mathrm{K}^{+}$media led to effective $\mathrm{Ca}^{2+}$ influx within $1.5 \mathrm{~min}$. Tracing the cell surface area of Acan $^{\text {tdT }}$-marked DS cells cultured on a soft substrate demonstrated functional contraction in high $\mathrm{K}^{+}$conditions in vitro (fig. S10B). Next, we tested whether the DS can functionally contract and compress microdissected intact hair follicles (Fig. 3A). Live imaging of freshly isolated follicles showed significant reduction of follicle widths after $3 \mathrm{~min}$ in high $\mathrm{K}^{+}$, consistent with contraction of concentric $\alpha \mathrm{SMA} /$ myosin rings (Fig. 3, B and D, and movie S1), which was effectively blocked by preincubation with the MLCK-specific inhibitor ML7 (Fig. 3, C and D, and movie S2). These data demonstrate that the DS can functionally contract by activation of voltage-gated $\mathrm{Ca}^{2+}$ channels in the $\mathrm{CaM} \rightarrow$ MLCK $\rightarrow$ MYL/MYH/aSMA pathway.

To determine whether smooth muscle-like contraction is a main function of the DS to propagate hair follicle regression in vivo, we blocked the MLCK pathway by topical ML7 application on the back skin of mice throughout catagen regression (Fig. 3E). In the back skins of mice receiving vehicle control, follicles regressed normally into telogen with the DP relocated next to the SC reservoir (Fig. $3 \mathrm{~F}$ and fig. S10C). Smooth muscle contraction blocking with ML7, however, resulted in a striking failure of regression with long stalled follicles stranded deep in the dermis in the center of the treated skin (Fig. 3, F and G), reminiscent of the DS-ablated phenotype (Fig. 1E). The DP here also remains at the tip of stalled follicles (Fig. $3 \mathrm{H}$ ), but in contrast to ablated follicles, the intact DS surrounds follicles including DP. Taken together, these data demonstrate that DS contraction is functionally required for follicle regression and DP niche relocation to the stem cells.

\section{Visualizing regression movements by intravital imaging} By utilizing intravital 2-photon microscopy of unperturbed skin in live mice $(15,18)$ (fig. S11A), we investigated where the DS contracts and by which force mechanism the DS drives niche relocation. For this we first established triple-color fluorescent reporters to visualize both the DS and neighboring progenitors (Fig. 4A). Acan ${ }^{t d T}$ labels the flat DS cell cytoplasm in red, and Tbx18 ${ }^{H 2 B G F P}$ (31) labels DS and DP nuclei in bright and dim green. To additionally label all nuclei of neighboring progenitors bright blue we generated a K14-H2BCer transgenic line (Fig. 4A and fig. S12, A and B). The triple-color fluorescent reporter combination (fig. S12C) enabled simultaneous live imaging of DS, DP, and follicle epithelium at a single excitation wavelength (fig. S11, B and C) and thus fine-mapping their absolute and relative movement over time.
Cell tracking during a 5-hour span of catagen regression demonstrated upward movement of the hair shaft and DP, as expected (Fig. 4B, fig. S13A, and movie S3). By contrast, the DS and ORS showed minimal movement in the long axis (Fig. 4C and fig. S13, B and C), indicating that hair shaft upward movement occurs at the interface between ORS progenitors and shaft, as previously observed (15). The lack of relative movement between DS and ORS suggested strong connections through the separating basement membrane. We next visualized hair shaft regression movements directly after contraction blocking in vivo (Fig. 4D). Live imaging the same follicle before and after topical ML7 application demonstrated that blocking smooth muscle contraction effectively abrogated upward movement of hair shafts. Together, our labeling setup and live imaging timeframe effectively captured the movements of key components during catagen regression and confirmed the requirement of smooth muscle contraction.

We then asked by which mechanism the niche becomes relocated during regression to reach its final position next to the stem cell reservoir and tested two long-standing hypotheses (24) (Fig. 4E): 1) an apoptotic force from the epithelial strand that pulls the DP (23) and 2) the DS contracts to push the DP and/or hair shaft upwards. To investigate the first hypothesis, we measured the length of the epithelial strand during the 5-hour imaging window. If shrinking due to dying cells would generate force for pulling the DP, we would expect shortening of the strand correlating to DP movement (Fig. 4E). However, we observed that individual epithelial strand lengths remained constant throughout (Fig. 4, F and G) despite steady upward DP movement, consistent with shaft and DP upward movement at similar rates (Fig. 4C). Although forces from individual migrating epithelial strand cells could contribute, our findings suggest that apoptotic force is not a major driver of regression.

To test the hypothesis that a DS-generated contractile force pushes the DP from underneath, we next investigated potential DS contraction movement below the DP. Coinciding with DP upward movement, we would expect DS centripetal movement underneath the DP. Therefore, we measured the cross-sectional diameter of the DS over time which, however, remained unchanged (Fig. 4, $\mathrm{H}$ and I) suggesting that direct DS contraction under the DP likely does not account for its relocation.

\section{Contraction at follicle bottleneck pulls the niche via epithelial strand}

We then considered a variation of the contraction hypothesis, in which DS contraction generates constriction forces that push the shaft upward, which in turn would pull both the epithelial strand and the DP upward (Fig. 5A). The observation of a constant epithelial strand length over time (Fig. 4, F 
and G) supports this alternative hypothesis in which the strand acts as a stable tether between the shaft and DP (Fig. $4 \mathrm{~F})$. Indeed, centripetal contraction movement of DS cells right at the border that forms a bottleneck between the shaftcontaining club hair and the narrower epithelial strand (Fig. $5, \mathrm{~B}$ and $\mathrm{C}$ ) indicates that DS contraction pushes the shaft upward (Fig. 5B and movie S3). Measuring at this "bottleneck" the cross-sectional diameter of the follicle-wrapping DS cell ring, we found a significant decrease over time coinciding with shaft upward movement (Fig. 5, D and E). These data reveal that the DS moves centripetally at the bottleneck toward the center suggesting that it contracts to generate the constriction force necessary for pushing the hair shaft upward - akin to the squeezing motion of a tooth paste tube which then pulls the DP by its connection to the hair shaft via the epithelial strand (Fig. 5F).

\section{Concluding remarks}

Here, we answer the long-standing question of how the niche is relocated nearly the entire length of the follicle to reach its essential stem cell-adjacent position. Through intravital imaging, contraction assays in isolated cells and intact follicles ex vivo, and in vivo inhibition of contraction, we have demonstrated that DS is a smooth muscle that contracts to power the key follicle regression movements during the catagen phase of the hair cycle. We have directly tested the hypotheses that proposed apoptotic or contraction forces propel catagen progression, based on observations possible at the time, and consolidated them into a model in which the niche relocates to the stem cells through a series of force relays (Fig. $5 \mathrm{~F})$. Having adapted the original "contraction hypothesis", the DS contracts throughout catagen at the club-strand bottleneck where, because of its angle, the centripetal constricting force gets redirected to an upward pushing force on the shaft. This force is then relayed through the tether-like epithelial strand pulling the DP. At the end stage of regression, when the hair shaft has reached its final position and the DS trails below the DP as a hollow sleeve, it is possible that forces generated by space reduction of apoptosing cells then pull in the niche next to the stem cell reservoir before launching regrowth in the next cycle that regenerates the follicle for new hair shaft production.

Intimate crosstalk between stem cells and their niches is vital for proper stem cell maintenance and cell fate decisions. Therefore, it is not surprising that niches are found in anatomically distinct locations for hematopoietic stem cells in the bone marrow and intestinal stem cells at the crypt base. Providing close proximities for paracrine signaling and insulation from outside influences, most stem cell niche systems under homeostasis remain structurally stable, but after injury to the niche, re-establishment is vital for the restoration of long-term function $(32,33)$. Although hematopoietic stem cells can mobilize and home to their niche, such as during transplantation (34), the more restricted freedom of movement of epithelial stem cells may limit their homing potential. Here we discover a smooth muscle function to relocate a niche to its stem cell reservoir to add to the vast array of diverse roles of smooth muscles throughout the body. This example highlights the evolutionary advantage of repurposing pre-existing functionality rather than inventing new systems in wake of new adaptive challenges. Further study may identify more instances of the function of smooth muscle contraction in regulating stem cell niche signaling range in homeostasis, which could be lost or exploited in disease.

\section{REFERENCES AND NOTES}

1. Y. Ge, E. Fuchs, Stretching the limits: From homeostasis to stem cell plasticity in wound healing and cancer. Nat. Rev. Genet. 19, 311-325 (2018). doi:10.1038/nrg.2018.9 Medline

2. P. Rompolas, K. R. Mesa, V. Greco, Spatial organization within a niche as a determinant of stem-cell fate. Nature 502, 513-518 (2013). doi:10.1038/nature12602 Medline

3. Y.-C. Hsu, L. Li, E. Fuchs, Transit-amplifying cells orchestrate stem cell activity and tissue regeneration. Cell 157, 935-949 (2014). doi:10.1016/j.cell.2014.02.057 Medline

4. E. Laurenti, B. Göttgens, From haematopoietic stem cells to complex differentiation landscapes. Nature 553, 418-426 (2018). doi:10.1038/nature25022 Medline

5. E. Legué, J.-F. Nicolas, Hair follicle renewal: Organization of stem cells in the matrix and the role of stereotyped lineages and behaviors. Development 132, 4143-4154 (2005). doi:10.1242/dev.01975 Medline

6. C. Clavel, L. Grisanti, R. Zemla, A. Rezza, R. Barros, R. Sennett, A. R. Mazloom, C.-Y. Chung, X. Cai, C.-L. Cai, L. Pevny, S. Nicolis, A. Ma'ayan, M. Rendl, Sox2 in the dermal papilla niche controls hair growth by fine-tuning BMP signaling in differentiating hair shaft progenitors. Dev. Cell 23, 981-994 (2012). doi:10.1016/j.devcel.2012.10.013 Medline

7. S. Müller-Röver, B. Handjiski, C. van der Veen, S. Eichmüller, K. Foitzik, I. A. McKay, K. S. Stenn, R. Paus, A comprehensive guide for the accurate classification of murine hair follicles in distinct hair cycle stages. J. Invest. Dermatol. 117, 3-15 (2001). doi:10.1046/j.0022-202x.2001.01377.x Medline

8. G. Cotsarelis, T. T. Sun, R. M. Lavker, Label-retaining cells reside in the bulge area of pilosebaceous unit: Implications for follicular stem cells, hair cycle, and skin carcinogenesis. Cell 61, 1329-1337 (1990). doi:10.1016/0092-8674(90)90696-C Medline

9. H. Oshima, A. Rochat, C. Kedzia, K. Kobayashi, Y. Barrandon, Morphogenesis and renewal of hair follicles from adult multipotent stem cells. Cell 104, 233-245 (2001). doi:10.1016/S0092-8674(01)00208-2 Medline

10. R. J. Morris, Y. Liu, L. Marles, Z. Yang, C. Trempus, S. Li, J. S. Lin, J. A. Sawicki, G. Cotsarelis, Capturing and profiling adult hair follicle stem cells. Nat. Biotechnol. 22, 411-417 (2004). doi:10.1038/nbt950 Medline

11. C. Blanpain, W. E. Lowry, A. Geoghegan, L. Polak, E. Fuchs, Self-renewal, multipotency, and the existence of two cell populations within an epithelial stem cell niche. Cell 118, 635-648 (2004). doi:10.1016/j.cell.2004.08.012 Medline

12. T. Tumbar, G. Guasch, V. Greco, C. Blanpain, W. E. Lowry, M. Rendl, E. Fuchs, Defining the epithelial stem cell niche in skin. Science 303, 359-363 (2004). doi:10.1126/science.1092436 Medline

13. G. Lindner, V. A. Botchkarev, N. V. Botchkareva, G. Ling, C. van der Veen, R. Paus, Analysis of apoptosis during hair follicle regression (catagen). Am. J. Pathol. 151 1601-1617 (1997). Medline

14. K. Foitzik, G. Lindner, S. Mueller-Roever, M. Maurer, N. Botchkareva, V. Botchkarev, B. Handjiski, M. Metz, T. Hibino, T. Soma, G. P. Dotto, R. Paus, Control of murine hair follicle regression (catagen) by TGF- $\beta 1$ in vivo. FASEB J. 14, 752760 (2000). doi:10.1096/fasebj.14.5.752 Medline

15. K. R. Mesa, P. Rompolas, G. Zito, P. Myung, T. Y. Sun, S. Brown, D. G. Gonzalez, K. B. Blagoev, A. M. Haberman, V. Greco, Niche-induced cell death and epithelial phagocytosis regulate hair follicle stem cell pool. Nature 522, 94-97 (2015). 
doi:10.1038/nature14306 Medline

16. M. Ito, G. Cotsarelis, K. Kizawa, K. Hamada, Hair follicle stem cells in the lower bulge form the secondary germ, a biochemically distinct but functionally equivalent progenitor cell population, at the termination of catagen. Differentiation 72, 548557 (2004). doi:10.1111/i.1432-0436.2004.07209008.x Medline

17. Y.-C. Hsu, H. A. Pasolli, E. Fuchs, Dynamics between stem cells, niche, and progeny in the hair follicle. Cell 144, 92-105 (2011). doi:10.1016/i.cell.2010.11.049 Medline

18. P. Rompolas, E. R. Deschene, G. Zito, D. G. Gonzalez, I. Saotome, A. M. Haberman, V. Greco, Live imaging of stem cell and progeny behaviour in physiological hairfollicle regeneration. Nature 487, 496-499 (2012). doi:10.1038/nature11218 Medline

19. V. Greco, T. Chen, M. Rendl, M. Schober, H. A. Pasolli, N. Stokes, J. Dela CruzRacelis, E. Fuchs, A two-step mechanism for stem cell activation during hair regeneration. Cell Stem Cell 4, 155-169 (2009). doi:10.1016/j.stem.2008.12.009 Medline

20. N. Oshimori, E. Fuchs, Paracrine TGF- $\beta$ signaling counterbalances BMP-mediated repression in hair follicle stem cell activation. Cell Stem Cell 10, 63-75 (2012). doi:10.1016/i.stem.2011.11.005 Medline

21. S. J. Mann, Hair loss and cyst formation in hairless and rhino mutant mice. Anat. Rec. 170, 485-499 (1971). doi:10.1002/ar.1091700409 Medline

22. W. Ahmad, M. Faiyaz ul Haque, V. Brancolini, H. C. Tsou, S. ul Haque, H. Lam, V. M. Aita, J. Owen, M. deBlaquiere, J. Frank, P. B. Cserhalmi-Friedman, A. Leask, J. A. McGrath, M. Peacocke, M. Ahmad, J. Ott, A. M. Christiano, Alopecia universalis associated with a mutation in the human hairless gene. Science 279, 720-724 (1998). doi:10.1126/science.279.5351.720 Medline

23. K. S. Stenn, R. Paus, Controls of hair follicle cycling. Physiol. Rev. 81, 449-494 (2001). doi:10.1152/physrev.2001.81.1.449 Medline

24. C. A. Jahoda, A. J. Reynolds, C. Chaponnier, J. C. Forester, G. Gabbiani, Smooth muscle alpha-actin is a marker for hair follicle dermis in vivo and in vitro. J. Cell Sci. 99, 627-636 (1991). Medline

25. S. Malgouries, S. Thibaut, B. A. Bernard, Proteoglycan expression patterns in human hair follicle. Br. J. Dermatol. 158, 234-242 (2008). doi:10.1111/j.13652133.2007.08339.x Medline

26. M. Rendl, L. Lewis, E. Fuchs, Molecular dissection of mesenchymal-epithelial interactions in the hair follicle. PLOS Biol. 3, e331 (2005). doi:10.1371/journal.pbio.0030331 Medline

27. A. Rezza, Z. Wang, R. Sennett, W. Qiao, D. Wang, N. Heitman, K. W. Mok, C. Clavel, R. Yi, P. Zandstra, A. Ma'ayan, M. Rendl, Signaling networks among stem cell precursors, transit-amplifying progenitors, and their niche in developing hair follicles. Cell Rep. 14, 3001-3018 (2016). doi:10.1016/j.celrep.2016.02.078 Medline

28. J. A. Beamish, P. He, K. Kottke-Marchant, R. E. Marchant, Molecular regulation of contractile smooth muscle cell phenotype: Implications for vascular tissue engineering. Tissue Eng. Part B Rev. 16, 467-491 (2010). doi:10.1089/ten.teb.2009.0630 Medline

29. M.-L. Bochaton-Piallat, G. Gabbiani, B. Hinz, The myofibroblast in wound healing and fibrosis: Answered and unanswered questions. F1000Res. 5, 752 (2016). doi:10.12688/f1000research.8190.1 Medline

30. R. A. Murphy, J. S. Walker, J. D. Strauss, Myosin isoforms and functional diversity in vertebrate smooth muscle. Comp. Biochem. Physiol. B Biochem. Mol. Biol. 117, 51-60 (1997). doi:10.1016/S0305-0491(96)00314-8 Medline

31. L. Grisanti, C. Clavel, X. Cai, A. Rezza, S.-Y. Tsai, R. Sennett, M. Mumau, C.-L. Cai, M. Rendl, Tbx18 targets dermal condensates for labeling, isolation, and gene ablation during embryonic hair follicle formation. J. Invest. Dermatol. 133, 344353 (2013). doi:10.1038/jid.2012.329 Medline

32. M. Dominici, V. Rasini, R. Bussolari, X. Chen, T. J. Hofmann, C. Spano, D. Bernabei, E. Veronesi, F. Bertoni, P. Paolucci, P. Conte, E. M. Horwitz, Restoration and reversible expansion of the osteoblastic hematopoietic stem cell niche after marrow radioablation. Blood 114, 2333-2343 (2009). doi:10.1182/blood-200810-183459 Medline

33. H. Bonig, T. Papayannopoulou, Hematopoietic stem cell mobilization: Updated conceptual renditions. Leukemia 27, 24-31 (2013). doi:10.1038/leu.2012.254 Medline

34. T. Lapidot, A. Dar, O. Kollet, How do stem cells find their way home? Blood 106 , 1901-1910 (2005). doi:10.1182/blood-2005-04-1417 Medline
35. P. Ellis, B. M. Fagan, S. T. Magness, S. Hutton, O. Taranova, S. Hayashi, A. McMahon, M. Rao, L. Pevny, SOX2, a persistent marker for multipotential neural stem cells derived from embryonic stem cells, the embryo or the adult. Dev. Neurosci. 26, 148-165 (2004). doi:10.1159/000082134 Medline

36. M. D. Muzumdar, B. Tasic, K. Miyamichi, L. Li, L. Luo, A global double-fluorescent Cre reporter mouse. Genesis 45, 593-605 (2007). doi:10.1002/dvg.20335 Medline

37. S. P. Henry, C.-W. Jang, J. M. Deng, Z. Zhang, R. R. Behringer, B. de Crombrugghe, Generation of aggrecan-CreERT2 knockin mice for inducible Cre activity in adult cartilage. Genesis 47, 805-814 (2009). doi:10.1002/dvg.20564 Medline

38. L. Madisen, T. A. Zwingman, S. M. Sunkin, S. W. Oh, H. A. Zariwala, H. Gu, L. L. Ng, R. D. Palmiter, M. J. Hawrylycz, A. R. Jones, E. S. Lein, H. Zeng, A robust and highthroughput Cre reporting and characterization system for the whole mouse brain. Nat. Neurosci. 13, 133-140 (2010). doi:10.1038/nn.2467 Medline

39. D. Voehringer, H.-E. Liang, R. M. Locksley, Homeostasis and effector function of lymphopenia-induced "memory-like" T cells in constitutively T cell-depleted mice. J. Immunol. 180, 4742-4753 (2008). doi:10.4049/jimmunol.180.7.4742 Medline

40. L. Li, D. D. Ginty, The structure and organization of lanceolate mechanosensory complexes at mouse hair follicles. elife 3, e01901 (2014). doi:10.7554/eLife.01901 Medline

41. C. Trapnell, L. Pachter, S. L. Salzberg, TopHat: Discovering splice junctions with RNA-Seq. Bioinformatics 25, 1105-1111 (2009). doi:10.1093/bioinformatics/btp120 Medline

42. B. Langmead, S. L. Salzberg, Fast gapped-read alignment with Bowtie 2. Nat. Methods 9, 357-359 (2012). doi:10.1038/nmeth.1923 Medline

43. C. Trapnell, B. A. Williams, G. Pertea, A. Mortazavi, G. Kwan, M. J. van Baren, S. L. Salzberg, B. J. Wold, L. Pachter, Transcript assembly and quantification by RNASeq reveals unannotated transcripts and isoform switching during cell differentiation. Nat. Biotechnol. 28, 511-515 (2010). doi:10.1038/nbt.1621 Medline

44. C. M. Pineda, S. Park, K. R. Mesa, M. Wolfel, D. G. Gonzalez, A. M. Haberman, P. Rompolas, V. Greco, Intravital imaging of hair follicle regeneration in the mouse. Nat. Protoc. 10, 1116-1130 (2015). doi:10.1038/nprot.2015.070 Medline

\section{ACKNOWLEDGMENTS}

We thank V. Horsley, T. Tumbar, R. Paus, M. Rangl, and R. Krauss for invaluable discussions and comments on the manuscript. We are especially grateful to $\mathrm{C}$. Jahoda for helpful discussions and launching dermal sheath research decades ago. Many thanks to D. Dubin and H. Khorasani for providing human scalp skin samples, and to the personnel at ISMMS Flow Cytometry, Microscopy, Genomics, and Mouse Genetics CoREs for technical assistance. Funding: The ISMMS Microscopy CoRE was supported by NIH Shared Instrumentation Grant IS10RR026639. N.H. and R.S. were supported by training grant T32GM007280 from NIH/NIGMS. N.H. was also supported by T32HHD075735 from NIH/NIDCR and F30AR070639 from NIH/NIAMS. D.S. and N.S. were supported by a fellowship of the Training Program in Stem Cell Research from the New York State Department of Health (NYSTEM-C32561GG). K.W.M was supported by The Science Appearance Career Development Award fellowship from the Dermatology Foundation. A.M. was supported by grants from NIH/OD (U54HL127624; U24CA224260). P.R. was supported by an NIH/NEl grant (R01EY030599). M.R. was supported by grants from NIH/NIAMS (R01AR071047; R01AR063151) and New York State Department of Health (NYSTEM-C029574 and NYSTEM-C32561GG), and a fellowship from the Irma T. Hirschl Trust. Author contributions: N.H., R.S. and M.R. designed the experiments and overall study. N.H. and M.R. wrote the manuscript. N.H., D.S., R.S., K.W.M., N.S., P.M. and L.G. performed the experiments. Z.W. and A.M assisted with transcriptome analysis. P.R. assisted with 2-photon imaging. All authors discussed results and participated in the manuscript preparation and editing. M.R. supervised the study. Competing interests: The authors declare no competing interests. $\mathrm{NH}, \mathrm{RS}$ and MR are inventors on a confidential provisional patent application that covers the subject matter of this article. Data and materials availability: All data presented in this study are available in the main text or the supplementary materials. Raw and analyzed RNA sequencing data generated during this study are available in the Gene Expression Omnibus (GEO) repository under accession GSE136996. 


\section{SUPPLEMENTARY MATERIALS}

science.sciencemag.org/cgi/content/full/science.aax9131/DC1

Materials and Methods

Figs. S1 to S13

Tables S1 to S3

References (35-44)

Movies S1 to S3

9 May 2019; accepted 11 December 2019

Published online 19 December 2019

10.1126/science.aax9131 
A

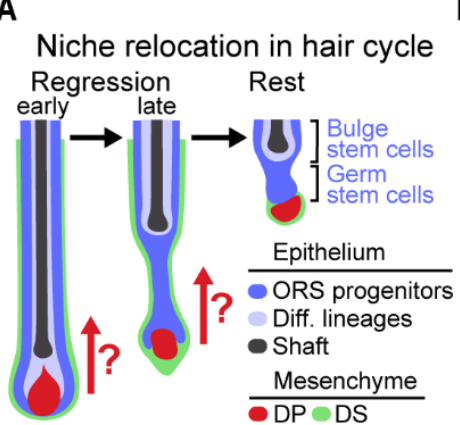

E

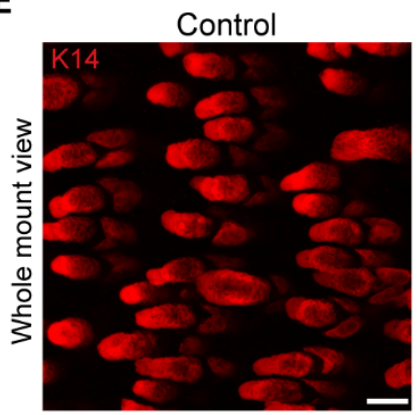

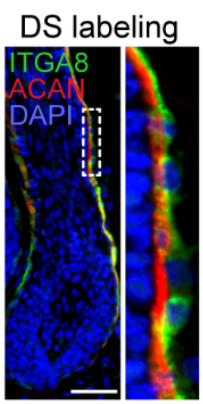

C

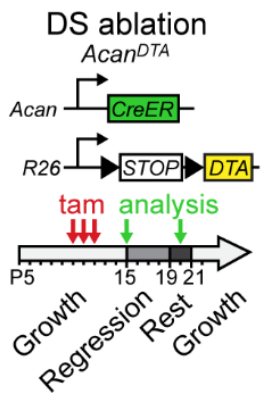

D

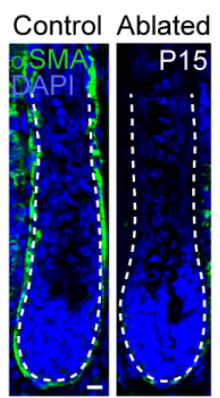

$\mathrm{F}$

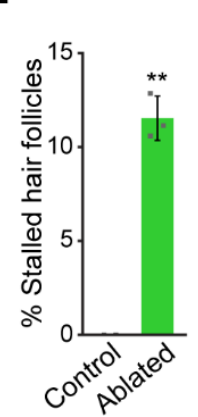

G

H

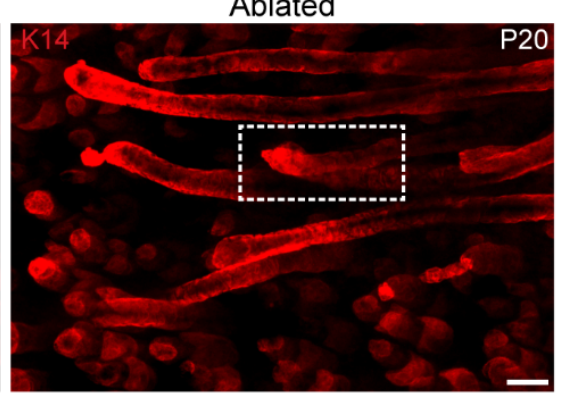

I
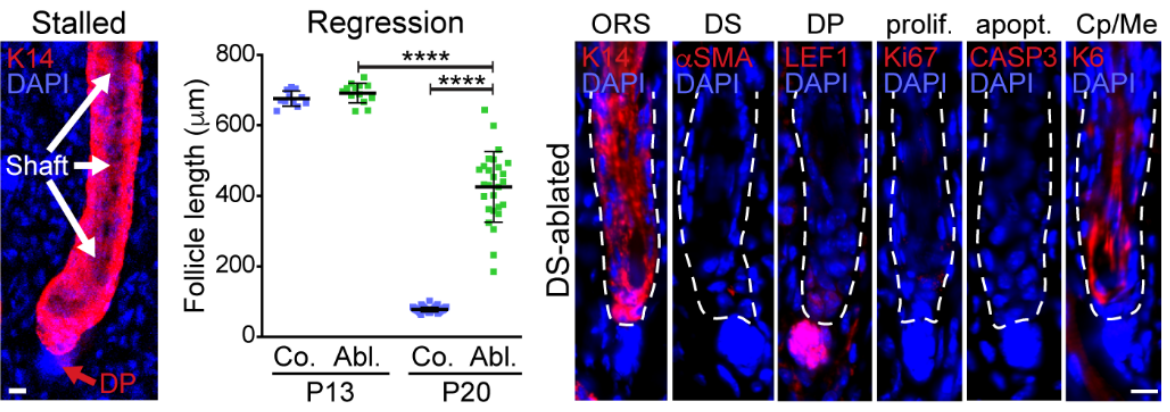

Fig. 1. The dermal sheath is required for hair follicle regression. (A) Schematic of catagen regression during the hair cycle. Hair shaft, inner root sheath (IRS) and matrix progenitors are eliminated by terminal differentiation and extrusion from the skin. The majority of outer root sheath (ORS) progenitors (blue) are eliminated by apoptosis. It is unknown how the surviving dermal papilla (DP) niche relocates to the bulge and germ stem cell reservoir to activate new hair growth after a period of rest. (B) Immunofluorescence (IF) for ACAN, secreted by ITGA8 ${ }^{+}$dermal sheath (DS) cells. (C) Schematic of cytotoxic DS ablation during regression. (D) IF for aSMA in control (R26 LSL-DTA) and DS-ablated (Acan ${ }^{\text {CreER; R26 }}$ LSL-DTA) back skin. (E) Whole mount IF for ORS marker K14 in P2O back skins (viewed from dermis side, anterior = left). Control follicles are in telogen resting phase. Note elongated follicles after DS ablation stalled in the regression phase. $(F)$ Quantification of \% stalled follicles at P20 ( $n=698$ in control and 895 in DS-ablated follicles in 5 mice). ${ }^{* *} P=0.003$, unpaired two-tailed t-test. $(G)$ Inset from $\mathrm{E}$ : hair shaft and DP remain at bulb tip of stalled follicles. $(\mathrm{H})$ Quantification of follicle lengths ( $n=11 \mathrm{P} 13$ control, $n=14$ P13 ablated, $n=80$ P20 control, $n=27$ P20 stalled; 11 mice). ${ }^{* * *} P<10^{-4}$, unpaired two-tailed $t$-test. (I) Stalled follicles have no DS ( $\left.\alpha S^{*} A^{-}\right)$, but retain intact $\mathrm{DP}\left(\mathrm{LEF} 1^{+}\right) . \mathrm{K} 14^{+}$ORS progenitor and $\mathrm{K}^{+}$companion $(\mathrm{Cp})$ layers are present and lack apoptosis (activated CASP3) or proliferation (Ki67) markers. Scale bars, $50 \mu \mathrm{m}[(\mathrm{B})$ and (E)] and 10 $\mu \mathrm{m}[(\mathrm{D})$ and $(\mathrm{I})]$. 
A

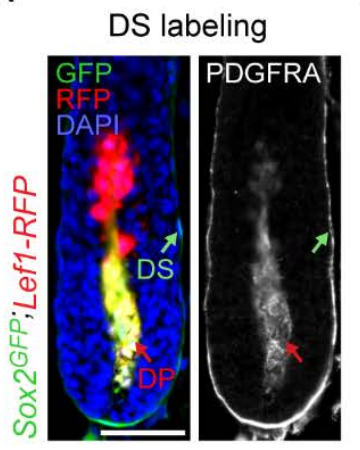

B DS isolation

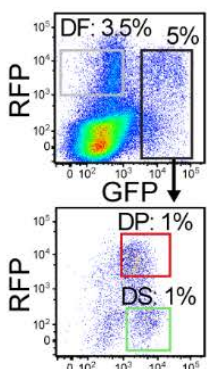

PDGFRA-BrV421
C

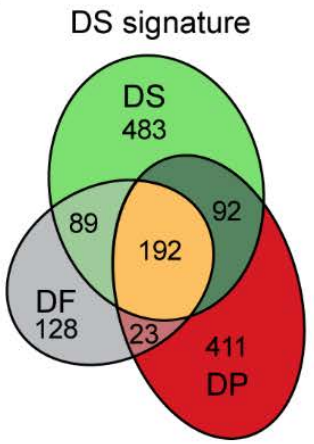

D

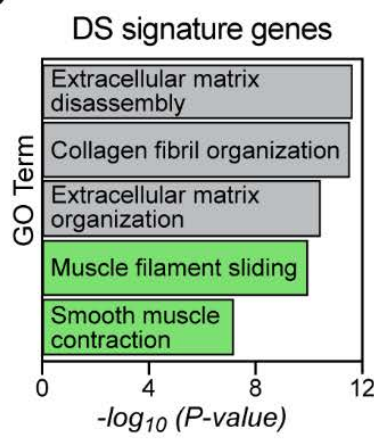

H

G
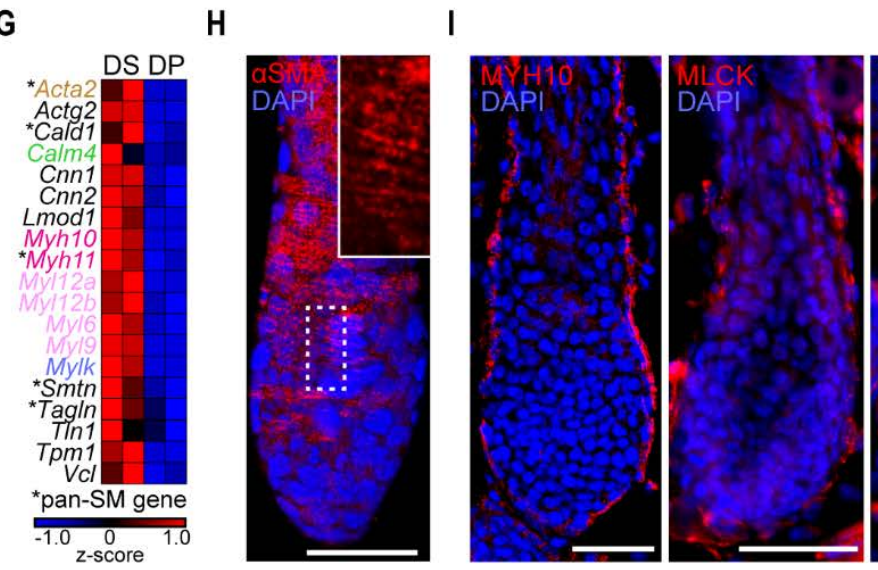

E

Smooth muscle GSEA
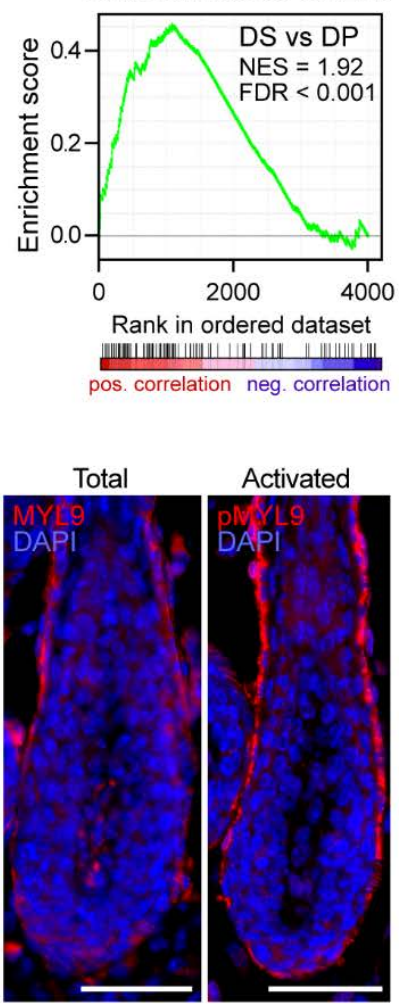

Activated

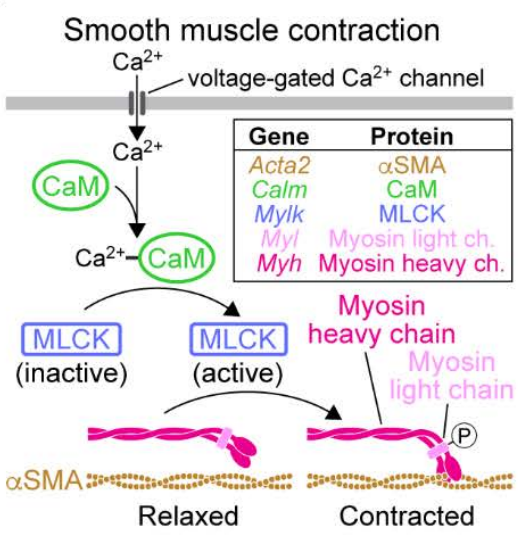

z-score

Fig. 2. The dermal sheath expresses the molecular machinery of smooth muscles. (A and $B)$ Flow cell sorting of DS and DP from Sox2 ${ }^{\text {GFP }}$;Lef1-RFP P5 back skin and IF for PDGFRA. Dermal fibroblasts (DF) were sorted for comparison. (C) Venn diagram of gene signatures. (D) Gene ontology analysis of DS signature. (E) Gene set enrichment analysis (GSEA) for genes involved in smooth muscle contraction and regulation are highly enriched in DS. (F) Schematic of $\mathrm{Ca}^{2+}-$ dependent smooth muscle contraction pathway. (G) Heatmap of smooth muscle contraction gene expression. $\mathrm{Ca}^{2+}$ contraction pathway and pan-smooth muscle genes (asterisks) are highly enriched in DS. (H) 3D IF for aSMA fibers arranged in a concentric ring-like network wrapping around the follicle. (I) IF of smooth muscle contraction components in DS. Scale bars, $50 \mu \mathrm{m}$. 
A

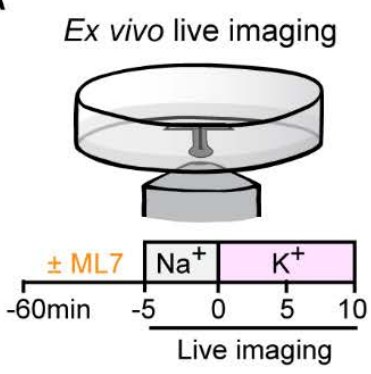

E

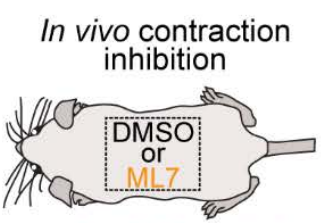

twice daily analysis

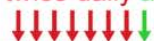

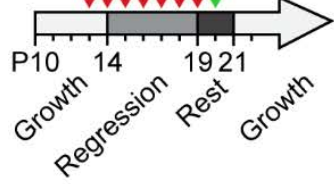

$\mathbf{F}$
B
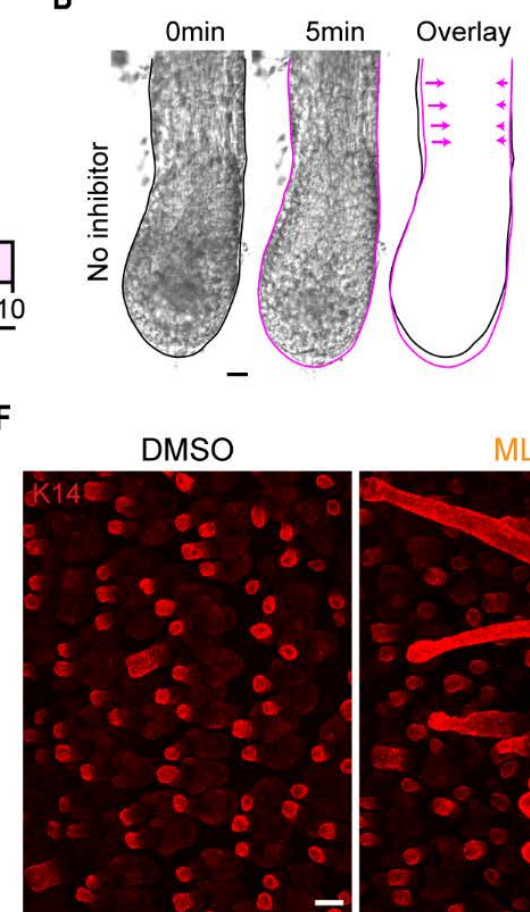

C
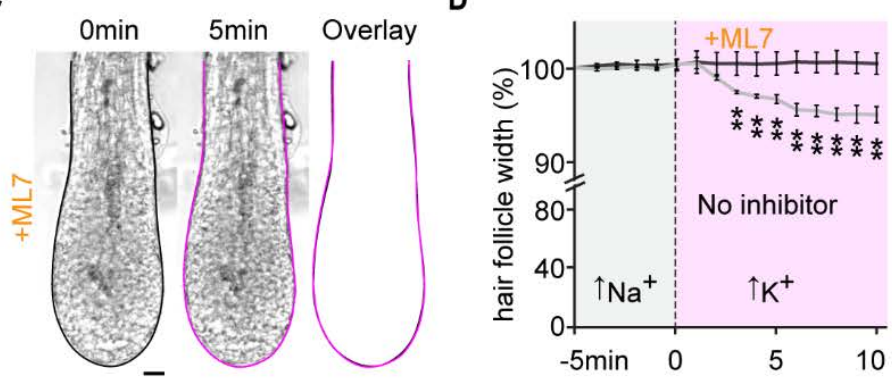

H

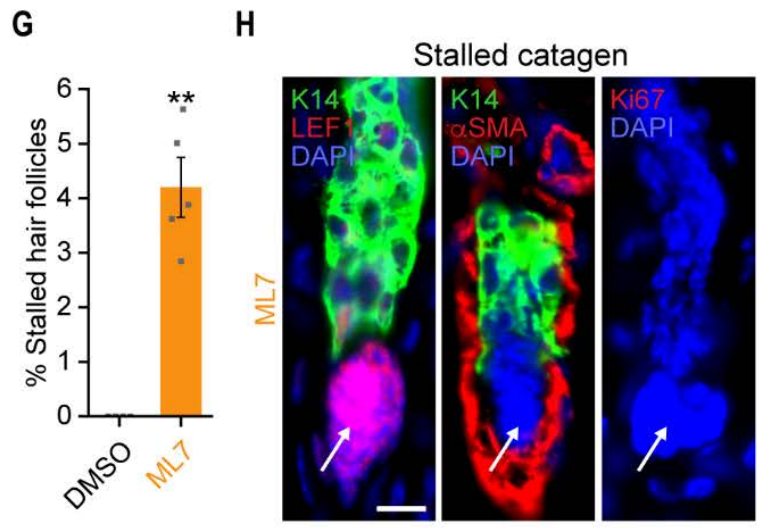

ML7

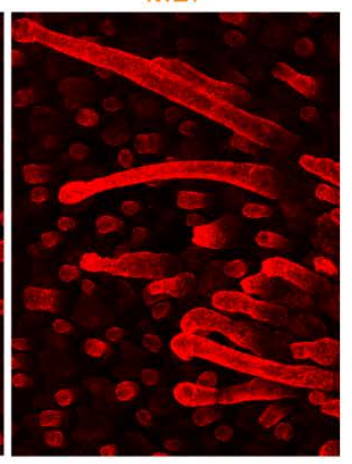

Fig. 3. The dermal sheath functionally contracts and is required for regression in vivo. (A) Schematic of live imaging microdissected follicles pre-incubated with or without MLCK inhibitor ML7 and after high $\mathrm{K}^{+}$depolarization. (B and C) Still images from brightfield movie at start (black) and end (pink) of high $\mathrm{K}^{+}$incubation. Overlays highlight reduction of follicle width, blocked by ML7. (D) Quantification of follicle widths during live imaging. $n=7$ follicles for ML7 and no inhibitor preincubation. Data points are mean $\pm \mathrm{s}$.d. ${ }^{* *} P<0.01$, unpaired two-tailed $t$-test. (E to G) Topical inhibition of MLCK by ML7 blocks hair follicle regression in vivo. Schematic of ML7 or vehicle application during catagen (E). Whole mount IF of P2O back skins show normal regression of follicles into telogen rest in control, but stalled follicles in contraction-inhibited ML7-treated regions (F). Quantification of \% stalled follicles ( $n=1071$ control, $n=1019$ ML7-treated; 10 mice) (G). Data bars are mean \pm s.d. ${ }^{* *} P=0.001$, unpaired two-tailed $t$-test. (H) IF for LEF1, Ki67, $\alpha$ SMA and K14. Stalled follicles have intact DS (aSMA) and DP (LEF1) that are no longer engulfed. Epithelial cells (K14) of stalled follicles are not proliferative (Ki67). Scale bars, $50 \mu \mathrm{m}[(\mathrm{B}),(\mathrm{C})$, and (F)] and $10 \mu \mathrm{m}(\mathrm{H})$. 
A

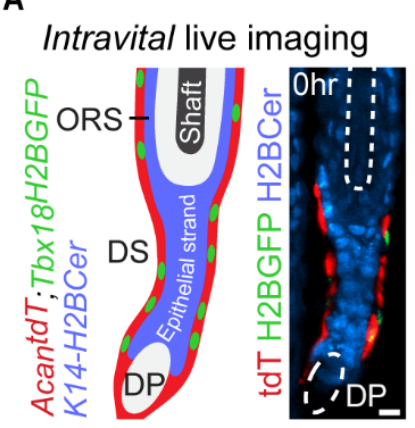

E

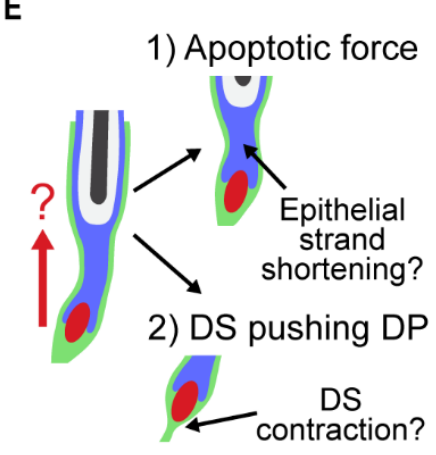

B

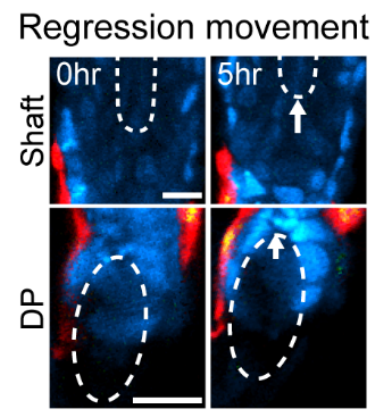

$F$

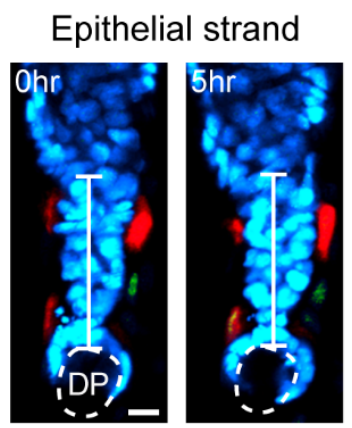

C

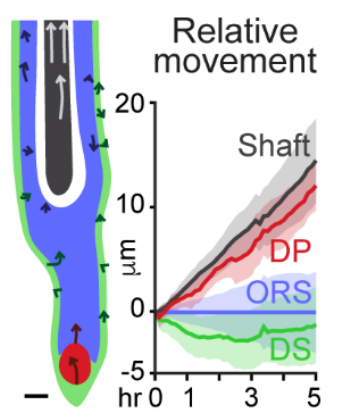

G

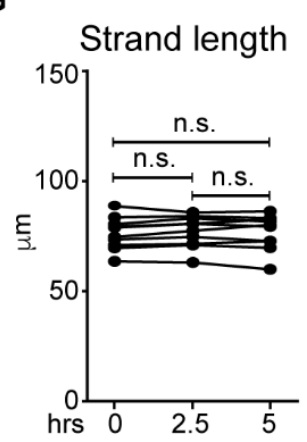

D

Contraction inhibition $\rightarrow$ movement
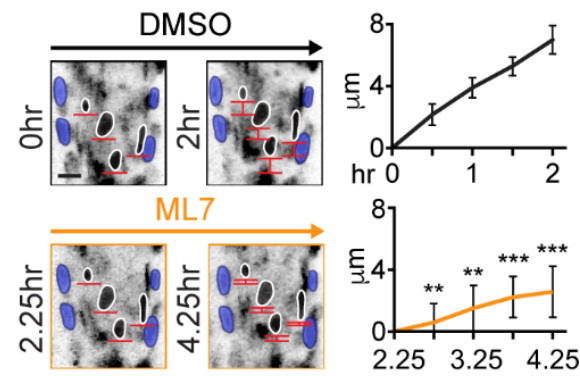

$\mathrm{H}$

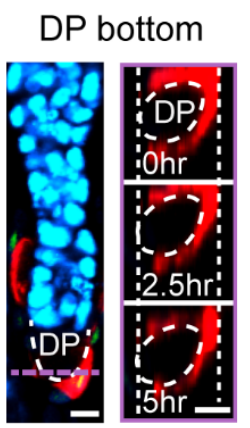

I

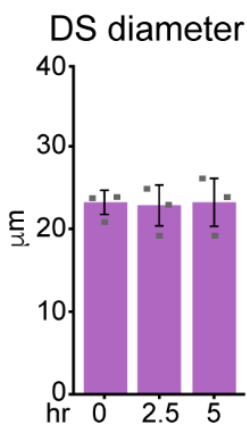

Fig. 4. Dermal sheath contraction is required for hair shaft and niche relocation. (A) Triplefluorescent reporter follicles for intravital 3D time-lapse imaging of catagen regression in live mice (5 hours). Acan tdT and Tbx18 ${ }^{\text {H2BGFP }}$ marks DS cytoplasm and nuclei, respectively. K14-H2BCer highlights all epithelial nuclei. The DP was recognized by low level Tbx18 ${ }^{\text {H2BGFP }}$ expression and surrounding epithelial and DS cells. (B) Upward movement of hair shaft and DP during regression. Tracking of individual ORS, shaft and DS cells and of upper and lower bounds of DP during 5-hour imaging. (C) Quantifications of live cell tracking relative to ORS movement (7.5 min intervals). Shaft and DP move upward relative to ORS and DS. Solid lines are average; shaded areas are s.d. $n=13$ HS, $n=8$ DP, $n=26$ ORS, and $n=17$ DS measurements (7 follicles, 3 imaging sessions). (D) In vivo contraction blocking during intravital imaging in K14-H2BCer mice. Inverted grayscale still images at beginning and end of vehicle control followed by ML7 application. Quantification of live tracking of shaft cells (black) relative to ORS (blue). $n=9$ follicles from 2 independent imaging sessions. ${ }^{*} P<$ 0.01 and ${ }^{* *} P<0.001$, paired two-tailed $t$-test. (E) Schematic of two historically hypothesized mechanisms for DP niche relocation during regression. ( $F$ and $G$ ) Fluorescence images from timelapse movie and quantification of the length of regressing epithelial strand. Strand lengths remain unchanged and stable ( $n=10$ follicles). $P=0.572$, unpaired two-tailed $t$-test ( 0 hours v. 5 hours). $(H)$ DS cross-sectional diameter at DP bottom $(n=4$ follicles). $P=0.994$, unpaired two-tailed $t$-test ( 0 hours v. 5 hours). (I) Quantification of DS diameter that remains unchanged over time. Data bars are mean \pm s.d. Scale bars, $10 \mu \mathrm{m}$. 
A

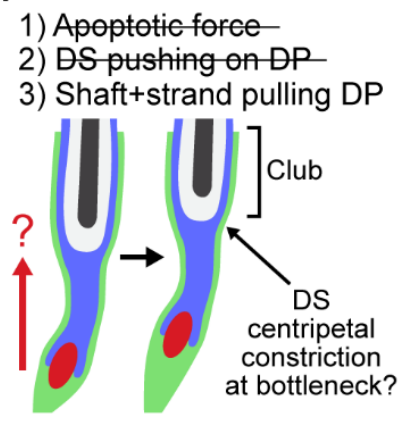

D

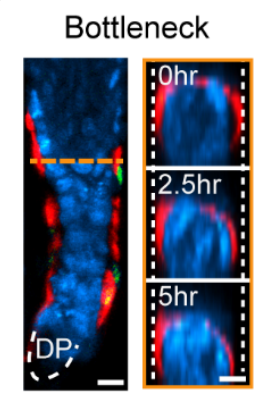

E
B

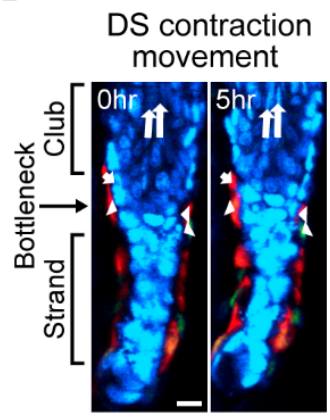

C

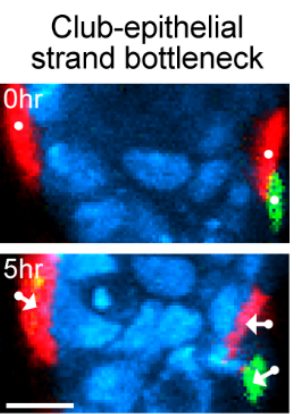

$F$ DP relocation by DS contraction
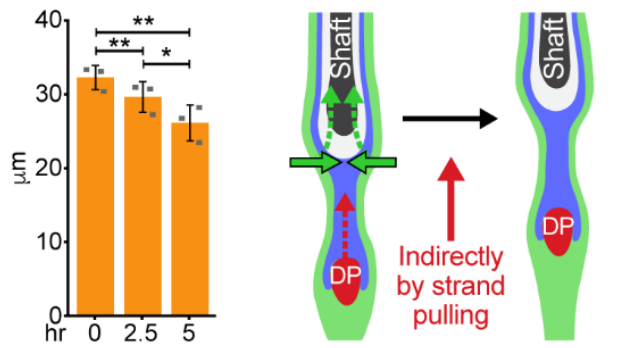

$\lessdot \underset{\text { contraction }}{\text { DS }}$

pushing

pulling
force

个 movement

Fig. 5. Dermal sheath contraction pushes the hair shaft and indirectly pulls the niche. (A) Schematic of alternative hypothesis for DP niche relocation by DS contraction at the bottleneck between the shaft-containing club hair and narrower regressing epithelial strand. (B) Live cell tracking of DS centripetal constriction movement at club-epithelial strand bottleneck and of hair shaft upward movement. Arrows are starting and ending positions of 5-hour tracking. (7 follicles, 3 imaging sessions). (C) High magnification of fluorescence time-lapse images at club-epithelial strand bottleneck. (D) DS cross-sectional diameter at club hair-epithelial strand bottleneck. The diameter of the follicle-wrapping DS cell ring decreased over time. (E) Quantification of DS diameter at bottleneck decreases over time ( $n=3$ follicles). ${ }^{*} P=0.021,{ }^{*} P=0.009$ and $P=0.010$, unpaired two-tailed $t$-test ( 0 hours v. 5 hours). (F) Model for DP niche relocation during regression. DS contraction forces centripetally constrict the follicle at the bottleneck to move the hair shaft upward, which pulls the DP upward via the epithelial strand. Data bars are mean \pm s.d. Scale bars, $10 \mu \mathrm{m}$. 